\title{
Maturation of White Matter is Associated with the Development of Cognitive Functions during Childhood
}

\author{
Zoltan Nagy, Helena Westerberg, and Torkel Klingberg
}

\begin{abstract}
In the human brain, myelination of axons continues until early adulthood and is thought to be important for the development of cognitive functions during childhood. We used diffusion tensor MR imaging and calculated fractional anisotropy, an indicator of myelination and axonal thickness, in children aged between 8 and 18 years. Development of working memory capacity was positively correlated with fractional anisotropy in two regions in the left frontal lobe, including a region between the superior frontal and parietal cortices.
\end{abstract}

\section{INTRODUCTION}

One of the most prolonged developmental processes in the human brain is the myelination of axons, which continues until the end of the second decade of life (Benes, 1989; Yakovlev \& Lecours, 1967). The longlasting development of the white matter is paralleled by the development of cognitive functions. Paul Flechsig (1920) was the first to demonstrate the regional differences in myelination rate and suggested that this may underlie the development of different functions. However, a direct correlation between the normal development of white matter structure and the improvement of cognitive functions has not been demonstrated.

In the present study, we address this question using diffusion tensor imaging (DTI), an MR technique that has the ability to reveal microstructural properties of white matter. This technique is based on the fact that the diffusion of water in the white matter of the brain is anisotropic (Moseley et al., 1990), so that it is faster along the axons than perpendicular to them. The axonal membrane itself induces some of this directional preference even without myelin (Gulani, Webb, Duncan, \& Lauterbur, 2001; Wimberger et al., 1995). However, myelination of the axons further increases the anisotropy as shown in studies comparing anisotropy with histological findings (Wimberger et al., 1995), comparing anisotropy in normal mice with that in knockout mice

Karolinska Institute, Astrid Lindgren Children's Hospital
Reading ability, on the other hand, was only correlated with fractional anisotropy in the left temporal lobe, in the same white matter region where adults with reading disability are known to have lower fractional anisotropy. Both the temporal and the frontal regions were also correlated with age. These results show that maturation of white matter is an important part of brain maturation during childhood, and that maturation of relatively restricted regions of white matter is correlated with development of specific cognitive functions.

lacking myelin (Gulani et al., 2001), as well as in human studies of demyelination (Werring, Clark, Barker, Thomson, \& Miller, 1999).

Conventional MR techniques have been used to measure a gradual increase in white matter volume during childhood (De Bellis et al., 2001; Giedd, Blumenthal, Jeffries, Castellanos, et al., 1999; Paus et al., 1999; Sowell, Thompson, Holmes, Jernigan, \& Toga, 1999; Caviness, Kennedy, Richelme, Rademacher, \& Filipek, 1996; Reiss, Abrams, Singer, Ross, \& Denckla, 1996; Pfefferbaum et al., 1994) based on the measurement of T1 signal. Although informative, these MR studies could not identify microstructural properties of white matter such as axonal directions. Previously, DTI has been used to find agerelated changes in anisotropy during childhood (Schmithorst, Wilke, Dardzinski, \& Holland, 2002; Mukherjee et al., 2001; Klingberg, Vaidya, Gabrieli, Moseley, \& Hedehus, 1999), but behavioral measures were not included in these studies.

We measured two different cognitive functions: visuospatial working memory (WM) and reading ability. Development of these functions continues throughout childhood but depend on different parts of the brain. By using two different cognitive functions we had the possibility to demonstrate some specificity of maturation (e.g., that maturation of a white matter region is more associated with development of one function and less with another). WM is most closely related to the function of the dorsolateral prefrontal cortex. The particular WM task we used (Figure 1) is based on a task that has previously been used to study the development of WM 
Figure 1. Schematic drawing of the visuospatial WM task.

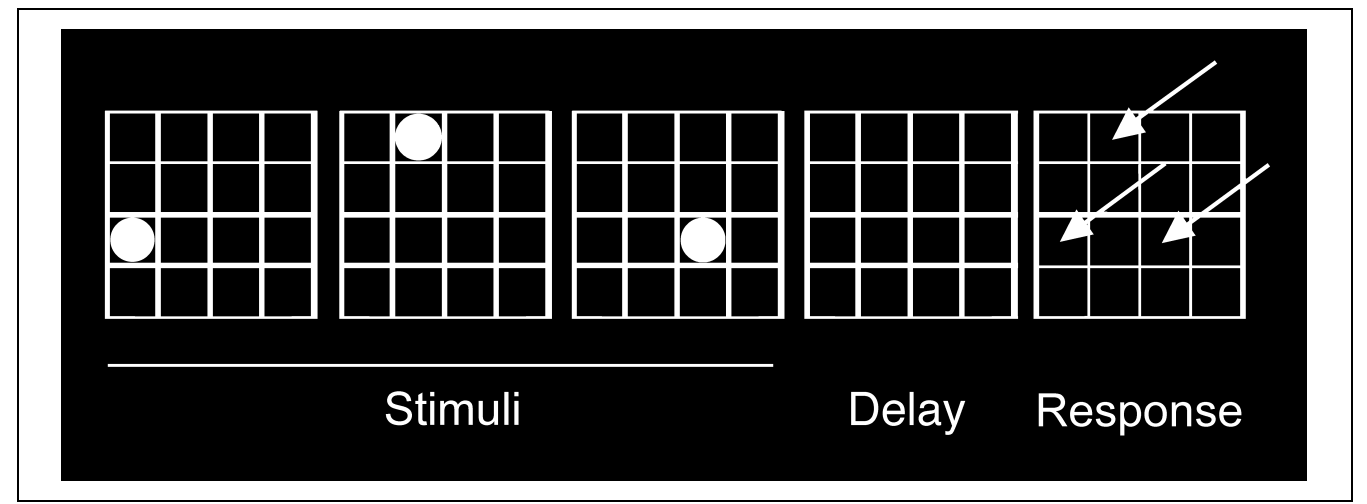

capacity in children (Westerberg, Hirvikoski, Forssberg, \& Klingberg, in press; Hale, Bronik, \& Fry, 1997). We have also used this task in a previous functional MRI study in children, in which development of WM capacity was found to correlate with increased cortical activity in the left superior frontal and left intraparietal cortices (Klingberg, Forssberg, \& Westerberg, 2002). A later study of development of WM confirmed these findings (Kwon, Reiss, \& Menon, 2002). In the present study, we thus hypothesized that we would find maturational changes in white matter close to these frontal and parietal regions, or in the white matter connecting them.

Development of reading ability is associated with changes in cortical activity in temporo-parietal, temporo-occipital, and ventral frontal regions (Turkeltaub, Gareau, Flowers, Zeffiro, \& Eden, 2003; Shaywitz et al., 2002). In a previous DTI study, it was found that reading ability, as measured by the word-ID task from the Woodcock test battery, correlated with white matter integrity in the left temporo-parietal region (Klingberg et al., 2000). In this region, adult subjects with reading disability had impaired integrity of white matter compared with controls. Furthermore, both within the reading impaired group and within the control group there was a positive correlation between reading ability and white matter structure. Given these results, we hypothesized that development of reading ability might be dependent on white matter structure in the temporo-parietal region. If such developmental trend could be detected, this might also provide insight into the possible mechanisms underlying developmental dyslexia.

Variability in white matter structure in a population of children could be divided into that related to differences in maturation, on the one hand, and interindividual variability on the other. Maturation can only partly be explained by the age of a child. Furthermore, even within the same individual, different cognitive functions could mature at different rates. A measure of cognitive function would thus provide a better measure of maturation than age. Our approach was to study children and adolescents (aged 8-18 years) with the underlying assumption that variability in cognitive function would mainly be determined by differences in maturation. In a separate analysis, we also used age as a covariate.

\section{RESULTS}

Twenty-three healthy, right-handed children (14 boys, 9 girls), aged 7.8-18.5 years (mean 11.9 years, SD 3.1), performed the reading and the WM tasks (Figure 1) and then underwent MR scanning. Fractional anisotropy (FA) was used as an index of white matter maturation. Analyses were conducted by correlating the behavioral variable with FA in each white matter voxel in the brain using SPM99 (Friston et al., 1995). When FA was correlated with WM scores, significant correlations $(p<.001)$ were found in two regions in the left frontal lobe (Figure 2A-B, Table 1), consistent with our hypothesis. Significant correlations were also found in the anterior corpus callosum, which connects the frontal lobes (Figure 2C). In addition, there was a correlation in the left temporo-occipital white matter (not shown), which was not predicted in our hypothesis. Reading time correlated negatively with FA values only in a single cluster, located in the left temporal lobe $(p<.001$, Figure 2D, Table 1).

In the analyses above, a stringent statistical threshold was used $(t>2.52$; cluster size $>230$ voxels; $p<.001)$. With a more lenient threshold $(t>2.52$; cluster size $>$ 120 voxels; $p<.05)$, there were two additional clusters that correlated with WM. These were located in the right superior fronto-parietal region $(x=15, y=-14, z=$ 62) and in the left occipito-temporal region $(x=-36$, $y=-70, z=2$ ).

FA values from the clusters in Table 1 were subsequently extracted from the most significant voxel in each cluster and plotted against the behavioral scores. The correlation was .54 for the superior fronto-parietal, .55 for the inferior frontal, .61 for the corpus callosum, .58 for the occipito-temporal, and .44 for the temporal cluster (Figure 3A-D). There were no significant differences in FA between boys and girls in any of the clusters 
related to WM (left superior fronto-parietal $p=.31$; left inferior frontal $p=.50$; corpus callosum $p=.29$; temporo-occipital $p=.09$ ) or reading ability (left temporal $p=.36$ ). Visual inspection suggested that one of the subjects in Figure 3D was an outlier. However, this was the youngest subject (7.8 years), and it was therefore to be expected that the child would also be the slowest reader. However, we made a confirmatory SPM analysis without this subject $(n=22)$, in which we still found a significant correlation between reading and FA in the same location $(p<.05)$. Furthermore, correlations between behavior and the extracted FA values were significant also using nonparametric methods (Spearman rank-correlation, $p<.001$ for each cluster).

The extent of fiber pathway organization can be estimated by coherence measures (Basser \& Pierpaoli, 1996). We measured the average angle between the direction of diffusion in a given voxel and the direction of diffusion in all its neighbors. Coherence images were calculated and normalized for each subject and statistical analyses identical to those done on the FA images were performed. However, these analyses did not result in any significant clusters that overlapped with the clusters of the FA analysis. With this method, there was

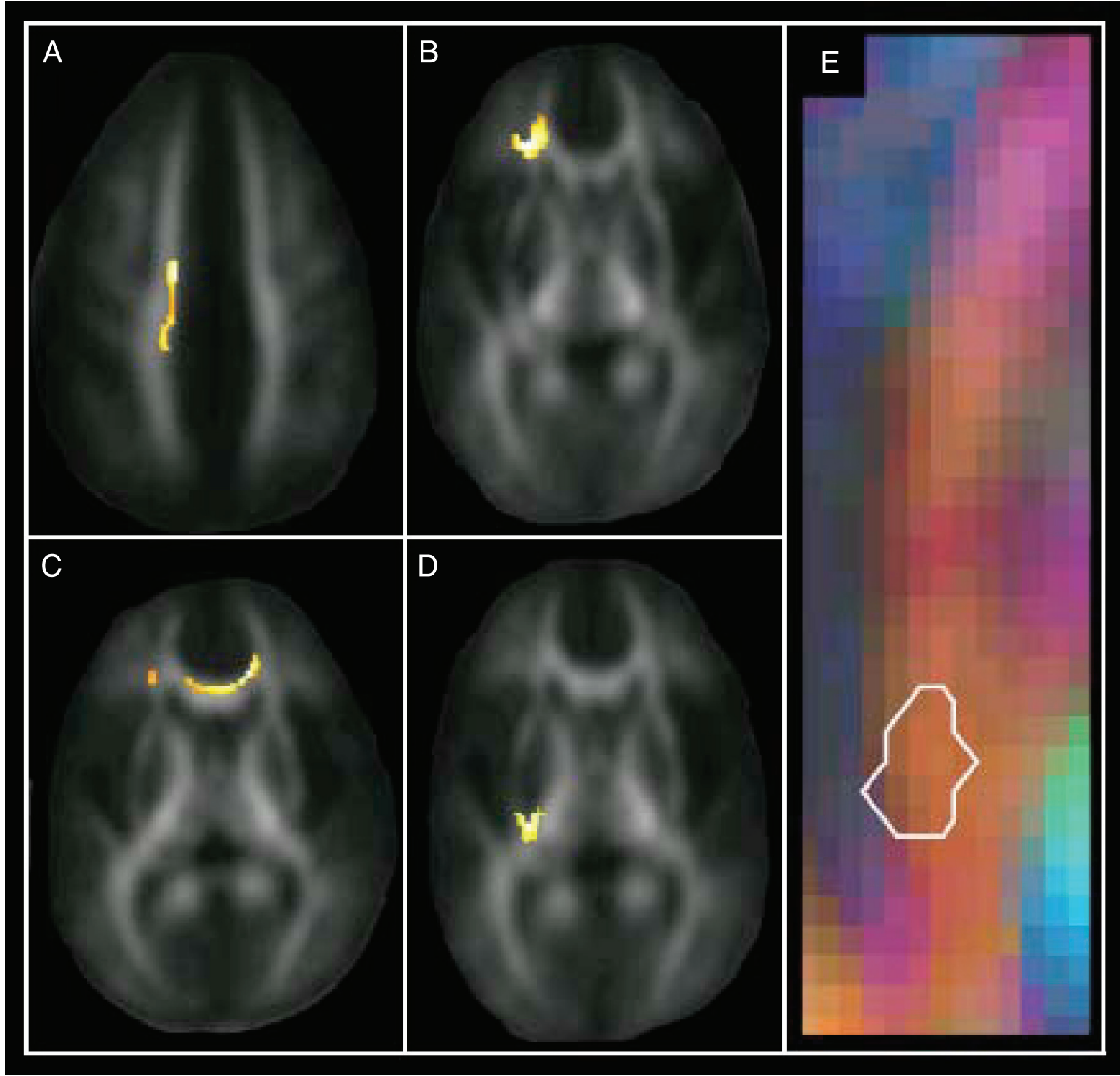

Figure 2. Regions of significant correlations between white matter anisotropy and cognitive performance. (A-C) Correlation between WM score and anisotropy. (D) Correlation between reading ability and anisotropy. The clusters are overlaid on the mean FA image from all 23 subjects, and each slice is through the most significant voxel of the cluster. (A) Left superior frontal lobe, extending into the parietal lobe. (B) Left inferior frontal lobe. (C) Genu of corpus callosum. (D) Left temporal lobe. (E) Main direction of diffusion in the region from (D). The colors red, blue, and green indicate anterior-posterior, left-right, and superior-inferior diffusion directions, respectively. The white lines mark the contour of the cluster correlated with reading ability. 
Table 1. Regions Where FA Values Correlated with Cognitive Test Scores

\begin{tabular}{|c|c|c|c|c|}
\hline Test & Significant Clusters & Corrected p Value & Cluster Size $\left(\mathrm{mm}^{3}\right)$ & Coordinates $(x, y, z)^{\mathrm{a}}$ \\
\hline \multirow[t]{4}{*}{ Working memory } & Superior fronto-parietal (L) & $<0.001$ & 1026 & $(-15,-22,54)$ \\
\hline & Inferior frontal $(\mathrm{L})^{*}$ & $<0.001$ & 1175 & $(-26,28,3)$ \\
\hline & Anterior corpus callosum* & $<0.001$ & 918 & $(14,27,9)$ \\
\hline & Temporo-occipital (L)* & $<0.001$ & 999 & $(-46,-48,0)$ \\
\hline Reading & Temporal (L) & $<0.001$ & 800 & $(-27,-28,6)$ \\
\hline
\end{tabular}

$\mathrm{L}=$ left.

${ }^{a}$ Talairach space in units of millimeters.

*Clusters with statistical significance after the effect of age is removed $(p<.05)$.

therefore no indication that fiber organization affected the correlation between FA and behavior.

\section{The Effect of Age}

In a separate analysis, we used age as a covariate in the WM and reading analyses, to see what correlations between cognitive test score and FA that remained significant after removing variability due to age. The regions that remained significant $(p<.05)$ are marked with an asterisk in Table 1.

\section{DISCUSSION}

In this study, we show that development of cognitive abilities during the later part of childhood is correlated with maturation of white matter. The improvement of
Figure 3. FA values and behavioral scores from the clusters shown in Figure 2A-D. Each point represents one individual.

(A) Left superior fronto-parietal.

(B) Left inferior frontal.

(C) Genu of corpus callosum.

(D) Left temporal lobe.
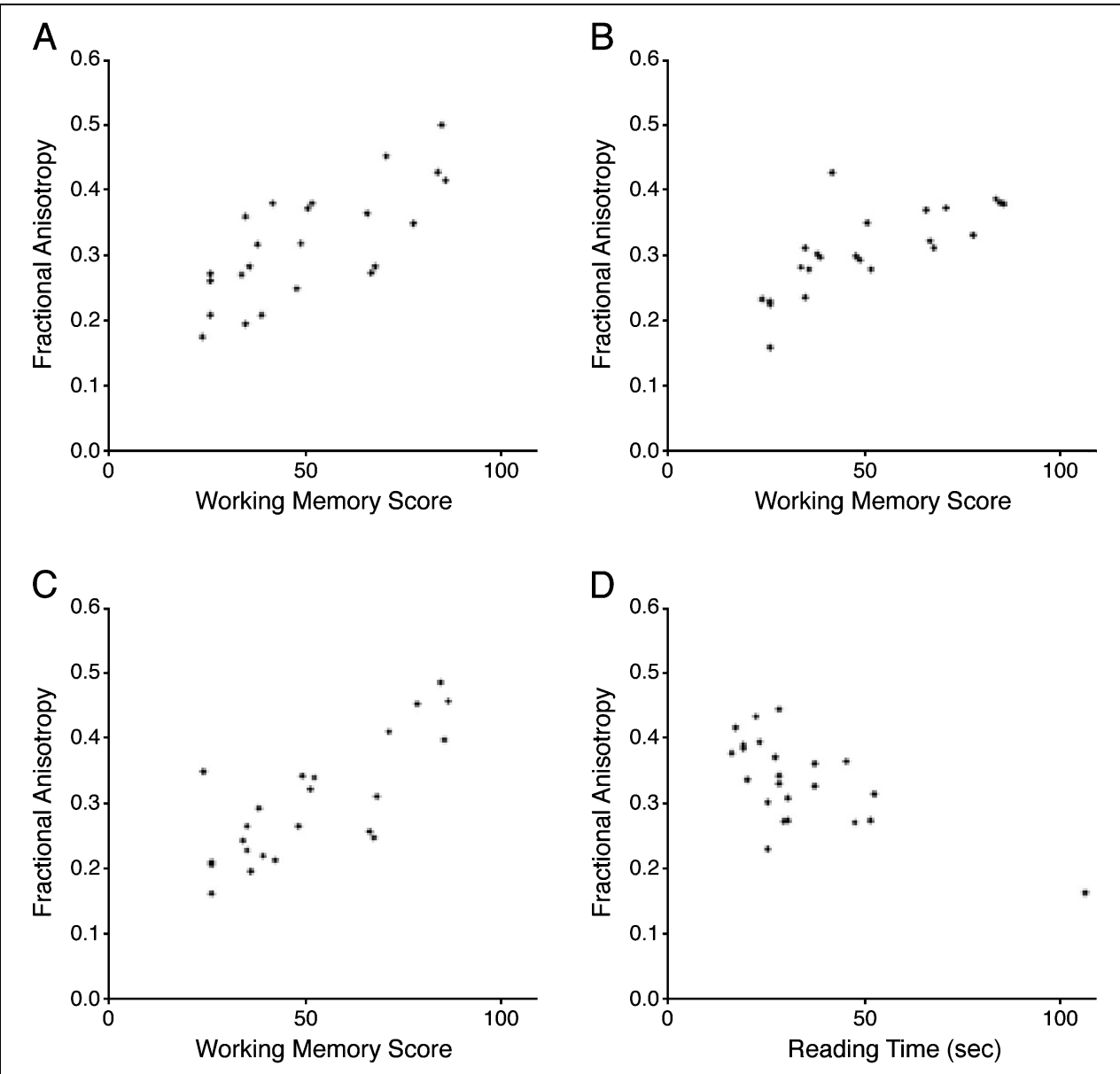
WM was associated with increased anisotropy in the superior and inferior left frontal lobe, whereas the development of reading ability only correlated with maturation of white matter in the left temporal lobe. The fact that white matter develops until late in childhood has been shown in previous studies (Schmithorst et al., 2002; De Bellis et al., 2001; Mukherjee et al., 2001; Giedd, Blumenthal, Jeffries, Castellanos, et al., 1999; Klingberg et al., 1999; Paus et al., 1999; Sowell et al., 1999; Caviness et al., 1996; Reiss et al., 1996; Pfefferbaum et al., 1994). The present study extends these previous findings by showing the regional and functional specificity of this maturation-with maturation of relatively restricted regions that are correlated with specific cognitive functions.

Within the left superior fronto-parietal cluster (WM correlation) and the left temporal white matter cluster (reading correlation), the correlation with FA was not significant after the effect of age was removed (Table 1). This shows that, for these regions, the relationship between behavior and FA is dependent on age, and therefore, actual childhood development. For other clusters (left inferior frontal, left occipito-temporal, corpus callosum), there remained a significant correlation between WM and FA also after removing the effect of age. This remaining correlation could be due to interindividual differences in WM capacity that are unrelated to maturation or to maturational differences that are not accounted for by age.

The reading ability cluster is located about $15 \mathrm{~mm}$ inferior and medial to the center of the region where an age-related change in T1-weighted signal was found previously (Paus et al., 1999). Furthermore, the reading ability cluster in the present study overlaps with the inferior part of a region where a previous DTI study found disturbances of white matter structure in adult subjects with reading disabilities (Klingberg et al., 2000). The cluster is located in a region where some axons run in an anterior-posterior direction (Figure 2E), which makes it possible that frontal and posterior language areas are connected by axons running through this region. The present result thus confirms the importance of this white matter region for reading ability, and extends the previous findings by demonstrating the importance of late maturation of white matter in this region. Knowledge of the normal development could indicate possible mechanisms in developmental disorders, such as developmental dyslexia. A disturbance of maturation in the temporal lobe would, for example, result in lower FA in adults. Future studies of development in children with and without reading problems might be able to answer these questions.

The regions where FA was correlated with WM capacity were mainly located within the frontal lobe. The white matter cluster in the superior part of the left frontal lobe is located about $10 \mathrm{~mm}$ medial and posterior to the cortical region in the superior frontal sulcus where development of visuospatial WM capacity and brain activity are correlated (Klingberg et al., 2002). It has also been shown that increased brain activity in the superior frontal cortex during childhood is correlated with improved performance on the Stroop task, a visual task requiring control of attention and response inhibition (Adleman et al., 2002). Furthermore, it was recently shown that the FA in the superior frontal lobe is directly correlated with brain activity in the superior frontal sulcus and intraparietal cortex in children (Olesen, Nagy, Westerberg, \& Klingberg, 2003). The structural maturation of white matter in the superior frontal lobe that was identified in the present study could thus be an underlying mechanism behind the increase in brain activity of the superior frontal cortex seen during development.

The correlation between FA and WM capacity in the inferior frontal lobe was expected based on the knowledge about the involvement of the frontal lobe in WM, but the connectivity of this white matter region is unclear. The maturation of white matter in the corpus callosum presumably improves the communication between the two frontal lobes. In the present study, FA in this region was significantly correlated with WM also after the effect of age was removed. Several prior studies have shown a correlation between white matter volume and age in the corpus callosum (De Bellis et al., 2001; Giedd, Blumenthal, Jeffries, Castellanos, et al., 1999). Consistent with the correlation to WM, there is evidence that the corpus callosum is affected in wide variety of childhood neuropsychiatric illnesses that involve impairments of WM and attention (Giedd, Blumenthal, Jeffries, Rajapakse, et al., 1999).

Anisotropy of diffusion in the white matter could theoretically reflect several histological characteristics in addition to myelination and axonal diameter, including the number of axons per cross-sectional area, and the coherence, that is, how well organized or parallel the fibers are. However, the coherence analysis did not indicate any differences in coherence that could explain the FA results, although this method only measures the coherence between voxels. The use of more diffusion directions might resolve the question of intravoxel coherence and its relation to FA (Frank, 2001). Because the number of axons does not increase after birth (LaMantia \& Rakic, 1990), the number of axons per cross-sectional area cannot explain the increase in FA during childhood. It is therefore more likely that the increase in FA is due to the increased diameter and myelination of axons during development. The amount of myelination and axonal thickness is directly related to each other (Waxman \& Sims, 1984), and these two phenomena are therefore difficult to separate in developmental studies. However, the physiological effects of increases in axon thickness and myelination are similar in that they both increase conduction speed. This could improve functionality by providing faster information transfer, and allowing a more precise timing in the 
communication between cortical areas. For the reading task, the crucial communication is presumably that between language areas in the temporo-parietal region and the inferior frontal cortex, although the connectivity is still unclear and could also include connections between posterior brain areas. Dyslexic subjects have a lower correlation of activity between posterior and anterior language areas (Horwitz, Rumsey, \& Donhue, 1998), which could be explained by disturbances in white matter connecting these regions (Klingberg et al., 2000). During development, there is also increased activity in this network (Turkeltaub et al., 2003), which could fit with the improved communication suggested by the present results. For the WM task, it is possible that higher conduction speed affects performance by facilitating recurrent excitation of cortical areas underlying active maintenance of visuospatial information. The improved communication could lead to a more stable network that is more resistant to interference and thus loss of information.

The present results show that the maturation of white matter affects cognitive functions. White matter maturation, in turn, may be affected by genetic pre-programming or by experience. There is evidence that activity in nerve cells promotes myelination of the axons (Stevens \& Fields, 2000). This provides a mechanism through which experience could affect myelination. It is still unclear, however, if these phenomena can be extended to the effect of experience on the human myelination to the degree that it can be detected by DTI.

\section{METHODS}

Tasks

In the WM task, the child was asked to remember the location of red circles that were presented sequentially in a $4 \times 4$ grid (Figure 1). After presentation, the child indicated the correct locations by pointing to an empty grid on a touch screen. The number of circles that the child should remember was gradually increased. When the child failed two attempts on a level, the testing was stopped. The total number of correctly remembered circles was summed up and used as the child's WM score. Only accuracy was of interest in this task. The subjects were not asked to respond as fast as possible and reaction times were not recorded.

During the reading task, the children read aloud a list of 20 Swedish words as fast as possible, without sacrificing accuracy. The time it took to read the words was taken as the reading variable that was used in the analysis. Shorter reading time indicates better reading ability.

\section{MR Scanning}

Diffusion-weighted scanning was performed with a 1.5-T General Electric Signa Echospeed scanner (Milwaukee,
WI, USA) with a single-shot, echo-planar imaging sequence, triggered with pulse gating with a delay of 300 msec. Diffusion was measured in 20 noncollinear directions $\left(b=1000 \mathrm{sec} / \mathrm{mm}^{2}\right)$. We also collected five non-diffusion-weighted images $\left(b=0 \mathrm{sec} / \mathrm{mm}^{2}\right)$. Eighteen slices were acquired from each subject, with an inplane resolution of $1.72 \times 1.72 \mathrm{~mm}$ and a slice thickness of $5 \mathrm{~mm}$. Eddy current distortions and head movements were corrected (Andersson \& Skare, 2002) before the calculation of diffusion tensors. In each voxel, FA was calculated (Basser \& Pierpaoli, 1996). FA images were smoothed with a 4-mm FWHM gaussian filter. From each subject, the mean of the five non-diffusion-weighted images was used to transform the anisotropy images to a common template in Talairach space, employing a set of linear and nonlinear transformation parameters, as implemented in the statistical software package SPM99 (Friston et al., 1995). Two statistical analyses were then performed, correlating FA in each voxel with WM or reading scores. We restricted the analysis to voxels with FA $>0.15$ to focus on white matter, which has higher FA values than gray matter and cerebrospinal fluid. The $p$ values in the SPM analyses were corrected for multiple comparison in the entire search volume, except in a second confirmatory analysis where one subject was excluded, in which there was a specific hypothesis about the location of the cluster.

\section{Acknowledgments}

We thank Jesper Andersson and Stefan Skare of the MR-Center at Karolinska Institute in Stockholm, Sweden. This work was supported by the Swedish Research Council.

Reprint requests should be sent to Torkel Klingberg, Karolinska Institute, Neuropediatric Unit, Astrid Lindgren Children's Hospital Q2:07, 17176 Stockholm, Sweden, or via e-mail: torkel.klingberg@kbh.ki.se.

\section{REFERENCES}

Adleman, N. E., Menon, V., Blasey, C. M., White, C. D., Warsofsky, I. S., Glover, G. H., \& Reiss, A. L. (2002). A developmental fMRI study of the Stroop color-word task. Neuroimage, 16, 61-75.

Andersson, J., \& Skare, S. (2002). A model-based method for retrospective correction of geometric distortions in diffusion-weighted EPI. Neuroimage, 16, 200-216.

Basser, P. J., \& Pierpaoli, C. (1996). Microstructural and physiological features of tissues elucidated by quantitative-diffusion-tensor MRI. Journal of Magnetic Resonance, Series B, 111, 209-219.

Benes, F. M. (1989). Myelination of cortical-hippocampal relays during late adolescence. Schizophrenia Bulletin, $15,585-593$.

Caviness, V. S., Kennedy, D. N., Richelme, C., Rademacher, J., \& Filipek, P. A. (1996). The human brain age 7-11 years: A volumetric analysis based on magnetic resonance images. Cerebral Cortex, 6, 726-736.

De Bellis, M. D., Keshavan, M. S., Beers, S. R., Hall, J., Frustaci, K., Masalehdan, A., Noll, J., \& Boring, A. M. (2001). Sex 
differences in brain maturation during childhood and adolescence. Cerebral Cortex, 11, 552-557.

Flechsig, P. (1920). Anatomie des Menschlichen Gebirns und Rückenmarks auf Myelogenetischer Grundlage. Leipzig: Thieme.

Frank, L. R. (2001). Anisotropy in high angular resolution diffusion-weighted MRI. Magnetic Resonance in Medicine, 45, 935-939.

Friston, K. J., Holmes, A. P., Worsley, K. J., Poline, J.-P., Frith, C. D., \& Frackowiak, R. S. J. (1995). Statistical parametric maps in functional imaging: A general linear approach. Human Brain Mapping, 2, 189-210.

Giedd, J. N., Blumenthal, J., Jeffries, N. O., Castellanos, F. X., Liu, H., Zijdenbos, A., Paus, T., Evans, A. C., \& Rapoport, J. L. (1999). Brain development during childhood and adolescence: A longitudinal MRI study. Nature Neuroscience, 2, 861-863.

Giedd, J. N., Blumenthal, J., Jeffries, N. O., Rajapakse, J. C., Vaituzis, A. C., Liu, H., Berry, Y. C., Tobin, M., Nelson, J., \& Castellanos, F. X. (1999). Development of the human corpus callosum during childhood and adolescence: A longitudinal MRI study. Progress in Neuro-psychopharmacology and Biological Psychiatry, 23, 571-588.

Gulani, V., Webb, A. G., Duncan, I. D., \& Lauterbur, P. C. (2001). Apparent diffusion tensor measurements in myelin-deficient rat spinal cords. Magnetic Resonance in Medicine, 45, 191-195.

Hale, S., Bronik, M. D., \& Fry, A. F. (1997). Verbal and spatial working memory in school-age children: Developmental differences in susceptibility to interference. Developmental Psychology, 33, 364-371.

Horwitz, B., Rumsey, J. M., \& Donhue, B. C. (1998). Functional connectivity of the angular gyrus in normal reading and dyslexia. Proceedings of the National Academy of Sciences, U.S.A., 95, 8939-8944.

Klingberg, T., Forssberg, H., \& Westerberg, H. (2002). Increased brain activity in frontal and parietal cortex underlies the development of visuo-spatial working memory capacity during childhood. Journal of Cognitive Neuroscience, 14, 1-10.

Klingberg, T., Hedehus, M., Temple, E., Salz, T., Gabrieli, J. D. E., Moseley, M. E., Poldrack, R. A. (2000). Microstructure of temporo-parietal white matter as a basis for reading ability: Evidence from diffusion tensor magnetic resonance imaging. Neuron, 25, 493-500.

Klingberg, T., Vaidya, C. J., Gabrieli, J. D. E., Moseley, M. E., \& Hedehus, M. (1999). Myelination and organization of the frontal white matter in children: A diffusion tensor MRI study. NeuroReport, 10, 1-5.

Kwon, H., Reiss, A. L., \& Menon, V. (2002). Neural basis of protracted developmental changes in visuo-spatial working memory. Proceedings of the National Academy of Sciences, U.S.A., 99, 13336-13341.

LaMantia, A. S., \& Rakic, P. (1990). Axon overproduction and elimination in the corpus callosum of the developing rhesus monkey. Journal of Neuroscience, 10, 2156-2175.

Moseley, M. E., Cohen, J., Kucharczyk, J., Mintorovitch, H. S., Asgari, M. F., Wendland, J., Tsuruda, J. \& Norman, D. (1990). Diffusion-weighted MR imaging of anisotropic water diffusion in cat central nervous system. Radiology, 176, 439-445.
Mukherjee, P., Miller, J. H., Shimony, J. S., Conturo, T. E., Lee, B. C., Almli, C. R., \& McKinstry, R. C. (2001). Normal brain maturation during childhood: Developmental trends characterized with diffusion-tensor MR imaging. Radiology, 221, 349-358.

Olesen, P., Nagy, Z., Westerberg, H., \& Klingberg, T. (2003). Combined analysis of DTI and fMRI data reveals a joint maturation of white and grey matter in a fronto-parietal network, Cognitive Brain Research, 18, 48-57.

Paus, T., Zijdenbos, A., Worsley, K., Collins, D. L., Blumenthal, J., Giedd, J. N., Rapoport, J. L., \& Evans, A. C. (1999). Structural maturation of neural pathways in children and adolescents: In vivo study. Science, 283, 1908-1911.

Pfefferbaum, A., Mathalon, D. H., Sullivan, E. V., Rawles, J. M., Zipursky, R. B., \& Lim, K. O. (1994). A quantitative magnetic resonance imaging study of changes in brain morphology from infancy to late adulthood. Archives of Neurology, 51, 874-887.

Reiss, A. L., Abrams, M. T., Singer, H. S., Ross, J. L., \& Denckla, M. B. (1996). Brain development, gender and IQ in children-A volumetric imaging study. Brain, 119, 1763-1774.

Schmithorst, V. J., Wilke, M., Dardzinski, B. J., \& Holland, S. K. (2002). Correlation of white matter diffusivity and anisotropy with age during childhood and adolescence: A cross-sectional diffusion-tensor MR imaging study. Radiology, 222, 212-218.

Shaywitz, B. A., Shaywitz, S. E., Pugh, K. R., Mencl, W. E., Fulbright, R. K., Skudlarski, P., Constable, R. T., Marchione, K. E., Fletcher, J. M., Lyon, G. R., \& Gore, J. C. (2002). Disruption of posterior brain systems for reading in children with developmental dyslexia. Biological Psychiatry, 52, 101-110.

Sowell, E. R., Thompson, P. M., Holmes, C. J., Jernigan, T. L., \& Toga, A. W. (1999). In vivo evidence for post-adolescent brain maturation in frontal and striatal regions.

Nature Neuroscience, 2, 859-861.

Stevens, B., \& Fields, R. D. (2000). Response of Schwann cells to action potentials in development. Science, 287, 2267-2271.

Turkeltaub, P. E., Gareau, L., Flowers, D. L., Zeffiro, T. A., \& Eden, G. F. (2003). Development of neural mechanisms for reading. Nature Neuroscience, 6, 767-773.

Waxman, S. G., \& Sims, T. J. (1984). Specificity in central myelination: Evidence for local regulation of myelin thickness. Brain Research, 292, 179-185.

Werring, D. J., Clark, C. A., Barker, G. J., Thomson, A. J., \& Miller, D. H. (1999). Diffusion tensor imaging of lesions and normal-appearing white matter in multiple sclerosis. Neurology, 52, 1626-1632.

Westerberg, H., Hirvikoski, T., Forssberg, H., \& Klingberg, T. (in press). Visuo-spatial working memory: A sensitive measurement of cognitive deficits in ADHD. Child Neuropsychology.

Wimberger, D. M., Roberts, T. P., Barkovich, A. J., Prayer, L. M. Moseley, M. E., \& Kucharczyk, J. (1995). Identification of "premyelination" by diffusion-weighted MRI. Journal of Computer Assisted Tomography, 19, 28-33.

Yakovlev, P. I., \& Lecours, A.-R. (1967). The myelogenetic cycles of regional maturation of the brain. In A. Minkowski (Ed.), Regional development of the brain in early life (pp. 3-65). Oxford: Blackwell Scientific Publications. 